\title{
Una mirada a los estereotipos de la violencia simbólica en los contextos educativos
}

\section{A look at the stereotypes of symbolic violence in educational contexts}

QUIJANO-VALLEJO, Bianca Carolina†*, HERNÁNDEZ-CUETO, Jaquelina Lizet, YAÑEZFLORES, Sara Margarita y SALINAS-AGUIRRE, María del Consuelo

Universidad Autónoma de Coahuila. Blvd. V. Carranza s/n. Col. República Oriente C.P.25280 Saltillo, Coah. México.

ID $1^{\mathrm{er}}$ Autor: Bianca Carolina, Quijano-Vallejo / ORC ID: 0000-0001-8488-2220, Researcher ID Thomson: X-3353-2019, PubMed Autor ID: f07dd0c9b2bef03c401f9b9045802c9df608

ID $1^{\text {er }}$ Coautor: Jaquelina Lizet, Hernández-Cueto / ORC ID: 0000-0002-3728-7434, Researcher ID Thomson: S-85882018, CVU CONACYT ID: 322702

ID $2^{\text {do }}$ Coautor: Sara Margarita, Yañez-Flores / ORC ID: 0000-0002-4750-4244, Researcher ID Thomson: S-9231-2018, CVU CONACYT ID: 352125

ID $3^{\text {er }}$ Coautor: María del Consuelo, Salinas-Aguirre / ORC ID: 0000-0002-6542-1813, Researcher ID Thomson: S-92442018, CVU CONACYT ID: 615335

\section{Resumen}

La presente investigación ofrece una visión de los temas más actuales desde la perspectiva de las figuras educativas respecto a los estereotipos que envuelven a la violencia simbólica, entre ellos la orientación vocacional o currículum, la sexualidad, el racismo, el nivel económico, el machismo y la violencia per se. Se observa cómo dentro de la sociedad actual dichos tópicos se tornan en tema de discusión, por lo que es importante conocer la realidad factual que ofrece la estadística para dilucidar cómo se presentan en las escuelas. El objetivo es conocer la realidad que circunscribe a la violencia simbólica en las escuelas. La metodología utilizada es cuantitativa en un nivel descriptivo con frecuencia y porcentaje para signalícticos y análisis univariable con tendencia central y estadígrafos de dispersión. La contribución es una mirada objetiva a la situación actual de la violencia simbólica en los contextos educativos, desde la perspectiva del profesorado.

\begin{abstract}
This research provides insight into the most current topics from the perspective of educational figures regarding stereotypes that surround symbolic violence, including vocational orientation or curriculum, sexuality, racism, economic level, machismo and violence per se. It is observed how within today's society these topics become a topic of discussion, so it is important to know the factual reality offered by statistics to determine how they are presented in schools. The aim is to know the reality that confines symbolic violence in schools. The methodology used is quantitative at a descriptive level frequently and percentage for signalitic and univariable analysis with central trend and dispersion statistics. The contribution is an objective look at the current situation of symbolic violence in educational contexts, from the perspective of teachers.
\end{abstract}

\footnotetext{
* Correspondencia del Autor (Correo electrónico: bianca.quijano@gmail.com)

$\dagger$ Investigador contribuyendo como primer autor.
} 


\section{Introducción}

El presente artículo ofrece una perspectiva diferente de la violencia simbólica dentro de los contextos educativos desde la perspectiva de los maestros, a través del análisis de seis variables complejas que pretenden operacionar a la violencia.

Para ello se llevó a cabo un análisis univariable que revela la tendencia de dichos tópicos dentro de los entornos escolares, el primero de ellos es sobre los estereotipos de la orientación vocacional o currículum, ya que hay carreras que de forma coloquial las personas circunscriben a un género determinado, por otra parte se encuentran los estereotipos de sexualidad, que dan una perspectiva sorprendente que difiere del discurso y de los resultados obtenidos, es decir; que se suele decir algo que convencionalmente sea aceptable, aunque prevalezca otro pensamiento sobre la sexualidad de los estudiantes.

La tercera variable mide el racismo y cómo es tipificado por las figuras educativas. También se mide la violencia simbólica a través del estereotipo del nivel económico y se revela cierta discriminación en la percepción de los respondientes.

El machismo como variable compleja ofreció mucha información de lo que piensan los maestros y de los roles establecidos.

Finalmente se mide la violencia per se que envuelve condiciones interesantes, ya que se indica en bajos niveles, pero se observa existente.

\section{Metodología a desarrollar}

Para la obtención de resultados se elaboró un documento ex profeso que permita identificar la violencia simbólica a través de las variables complejas orientación vocacional o currículum, sexualidad, racismo, nivel económico, machismo y violencia. Dicho instrumento se validó a través de un análisis de Cronbach.

Se desarrolló un análisis univariable de tendencia central y estadígrafos de dispersión con identificación de media de medias. Para conocer las características de la muestra extractada se hizo un análisis de frecuencia y porcentaje en signalícticos.

\section{Resultados}

En el presente apartado se muestran los resultados extraídos de los análisis estadísticos referentes a la manifestación de la violencia simbólica a través de un estudio univariable de las variables complejas orientación vocacional o currículum, sexualidad, racismo, nivel económico, machismo y violencia.

\section{Pruebas de confiabilidad y consistencia interna}

Para establecer la confiabilidad y validez del instrumento se realizó el análisis de Alfa de Cronbach, obteniéndose un alfa de 0.95 lo cual indica que según el criterio de Nunnaly \& Berstein (1994) es viable; ya que establecen que de 0.7 para investigaciones exploratorias, 0.80 para investigación básica, 0.90 para toma de decisiones $\mathrm{y}, \quad 0.95$ para investigaciones aplicadas.

No hay redundancia, ya que las variables no presentan valores superiores o iguales a 0.95 en la columna de correlación total entre los ítems. Aunado a este análisis también se llevó a cabo una revisión por expertos, de donde se tomaron en consideración sus opiniones objetivas y fundamentadas para la elaboración del instrumento.

\section{Resultados de frecuencia y porcentaje de signalícticos}

Con respecto al sexo de los sujetos se observa que el $59.42 \%$ son del sexo femenino y el $40.57 \%$ son del sexo masculino. La oscilación de la edad está entre los 21 y 67 años de edad, teniendo más personas en un rango de 25 a 35 años. Con un grado de estudios del $51.22 \%$ de licenciatura y en el caso del mismo grado de estudios, pero con un título de ingeniería el $12,29 \%$ seguido con un $28 \%$ con maestría, sólo un $2.45 \%$ con doctorado. Respecto al tipo de institución donde laboran un $36.88 \%$ es de escuela privada, $31.96 \%$ es de escuela pública y $31.14 \%$ en escuela católica-religiosa. Sobre el área en la que imparte clases el $31.14 \%$ es del área de humanidades, el $11.47 \%$ es del área de ciencias sociales, el $15.98 \%$ es del área de experimentales, el $16.80 \%$ es del área de comunicación y, un $24.59 \%$ del área de matemáticas. 
Sobre la orientación sexual de los sujetos, el $98.36 \%$ es heterosexual, un 1.22 es homosexual y un $0.40 \%$ es bisexual. Sobre la religión, el $82.78 \%$ manifestaron que son católicos.

\section{Resultados Univariables de Violencia Simbólica}

\section{Análisis Univariable de Estereotipos por orientación vocacional o currículum}

La orientación vocacional fue medida a través de estereotipos sobre el género en esta área.

Con base en el análisis de media de medias se observa que los sujetos indican que mayormente una mujer que tiene uno o más hijos debería trabajar menos y una mujer debe estudiar carreras que les permitan educar a sus hijos. De forma regular mencionan que es común que los homosexuales estudien carreras diseñadas para mujeres, es común que las lesbianas estudien carreras diseñadas para hombres y los hombres deben estudiar carreras que les permitan ganar más dinero que las mujeres.

En menor proporción mencionan que los hombres deben estudiar carreras diseñadas para ellos, las mujeres deben estudiar carreras diseñadas para ellas, es más importante que los hombres terminen la universidad que las mujeres y que la vocación de las mujeres es dedicarse al cuidado del hogar.

\begin{tabular}{|l|c|c|r|r|r|}
\hline \multicolumn{1}{|c}{$\mathbf{n = 2 4 4}$} & $\overline{\mathbf{X}}$ & $\mathbf{S k}$ & $\mathbf{K}$ & $\mathbf{C V}$ & $\mathbf{Z}$ \\
\hline Trabajar menos & 3.86 & 0.11 & -1.35 & 87 & 1.1 \\
\hline M.Carr edu hijos & 3.51 & 0.50 & -1.04 & 100 & 1 \\
\hline Ho. carr de M. & 2.53 & 0.90 & -0.51 & 129 & 0.8 \\
\hline Les. carr de H. & 2.29 & 0.97 & -0.34 & 133 & 0.8 \\
\hline H.ganar + din & 2.10 & 1.00 & -0.44 & 139 & 0.7 \\
\hline H.Carr p ellos & 2.02 & 1.22 & 0.24 & 148 & 0.7 \\
\hline M.Carr p ellas & 2.01 & 1.22 & 0.29 & 146 & 0.7 \\
\hline Más imp. Est H. & 1.72 & 1.41 & 0.78 & 166 & 0.6 \\
\hline Voca hogar & 1.34 & 1.69 & 1.87 & 183 & 0.5 \\
\hline$\overline{X X}=2.38$ Ss=0.3 LS=2.68 LI $=2.08$ \\
\hline
\end{tabular}

Tabla 1 Análisis univariable de orientación vocacional o currículum

Fuente: elaboración propia

Con respecto a sesgo, los valores se inclinan al positivo, lo que indica que tienen tendencia a la parte baja de la escala. La representación icónica de las variables indica que son platicúrticas $(K<3)$, con tendencia de colas más anchas en la representación de la normal.
De acuerdo al puntaje Z (>1.96) se lee que la tendencia es a no extrapolar, ya que tienen alta variabilidad $(Z<1.96)$ en sus respuestas.

\section{Análisis Univariable de Estereotipos de sexualidad}

La sexualidad fue medida a través de los prejuicios sobre la sexualidad de hombres y mujeres. Con base en el análisis de media de medias se observa que los sujetos indican que mayormente el sexo es exclusivo para personas adultas, el hombre debe tener sexo antes del matrimonio y la mujer debe tener sexo antes del matrimonio.

De forma regular el hombre necesita más del sexo que las mujeres, los hombres y las mujeres no pueden ser amigos porque siempre hay atracción sexual, las relaciones sexuales son para las personas casadas, es normal que el hombre engañe a su pareja, los hombres deben tener más experiencias sexuales que las mujeres, las mujeres deben llegar vírgenes al matrimonio y es responsabilidad exclusiva de la mujer usar un método anticonceptivo para evitar embarazos. En menor proporción, es normal que la mujer engañe a su pareja, la vida sexual de un varón debe iniciar antes que la de mujeres y es común que la mujer tolere la infidelidad de su pareja.

\begin{tabular}{|l|r|r|r|r|c|}
\multicolumn{1}{|c|}{$\mathbf{n = 2 4 4}$} & $\overline{\mathbf{X}}$ & \multicolumn{1}{c}{ Sk } & \multicolumn{1}{c|}{$\mathbf{K}$} & $\mathbf{C V}$ & $\mathbf{Z}$ \\
\hline Sex.Adultos & 3.95 & 0.37 & -1.48 & 102 & 0.98 \\
\hline H.sex an matri & 2.53 & 1.03 & -0.35 & 138 & 0.73 \\
\hline M.sex ant matri & 2.52 & 1.07 & -0.19 & 136 & 0.73 \\
\hline H.nec más sexo & 2.20 & 1.08 & -0.17 & 142 & 0.71 \\
\hline HyM.Amigos & 2.14 & 1.02 & -0.22 & 134 & 0.74 \\
\hline Rel.sex casadas & 1.90 & 1.47 & 0.84 & 165 & 0.61 \\
\hline H.engañe & 1.48 & 1.66 & 1.54 & 182 & 0.55 \\
\hline H.ex. Sex. M. & 1.41 & 1.54 & 1.18 & 176 & 0.57 \\
\hline Virgen & 1.34 & 1.83 & 2.48 & 188 & 0.53 \\
\hline M.mét antic & 1.19 & 2.06 & 3.25 & 212 & 0.47 \\
\hline M.engañe & 1.09 & 2.07 & 3.33 & 211 & 0.47 \\
\hline Vida sexual & 0.90 & 2.14 & 3.56 & 221 & 0.45 \\
\hline M.tol infid & 0.67 & 2.91 & 7.90 & 277 & 0.36 \\
\hline$\overline{X X}=1.79 \quad$ Ss=0.6 LS=2.39 LI=1.19 \\
\hline \multicolumn{5}{|c|}{ LS $=1.5$} \\
\hline
\end{tabular}

Tabla 2 Análisis univariable de estereotipo de sexualidad Fuente: elaboración propia

Con respecto a sesgo, los valores se inclinan al positivo, lo que indica que tienen tendencia a la parte baja de la escala. 
La representación icónica de las variables indica que son platicúrticas, con tendencia de colas más anchas en la representación de la normal, sin embargo, del análisis se pueden observar 4 datos con tendencia leptocúrtica $(K>3)$, los cuales son: es responsabilidad exclusiva de la mujer usar un método anticonceptivo para evitar embarazos, es normal que la mujer engañe a su pareja, la vida sexual de un varón debe iniciar antes que la de mujeres y la mujer debe tolerar las infidelidades de su pareja. De acuerdo al puntaje Z (>1.96) se lee que la tendencia es a no extrapolar, ya que tienen alta variabilidad $(\mathrm{K}<1.96)$ en sus respuestas.

\section{Análisis Univariable de Racismo}

El color de piel fue medido a través de la forma en que mayormente la sociedad tiene prejuicios respecto a este tema. Con base en el análisis de media de medias, se observa que los sujetos indican que mayormente es normal referirse como "morenito" a una persona de piel morena $\mathrm{y}$ es normal referirse a una persona de piel morena como prieto. De forma regular, es más fácil triunfar en la vida si tu color de piel es claro, es común poner apodos a las personas por su color de piel, es normal referirse a una persona de piel morena como indito, y es gracioso poner apodos a las personas por su color de piel.

En menor proporción la gente de piel morena es más violenta que la gente de piel clara, es normal insultar a alguien en la calle por su color de piel y las personas de tez clara son más inteligentes que la gente de tez morena. El color de piel fue medido a través de la forma en que mayormente la sociedad tiene prejuicios respecto a este tema.

\begin{tabular}{|l|c|c|r|r|c|}
\hline \multicolumn{1}{|c}{$\mathbf{n = 2 4 4}$} & $\overline{\mathbf{X}}$ & \multicolumn{1}{c}{ Sk } & \multicolumn{1}{c|}{ K } & \multicolumn{1}{c|}{$\mathbf{C V}$} & $\mathbf{Z}$ \\
\hline Morenito & 3.34 & 0.58 & -1.10 & 109 & 0.92 \\
\hline Prieto & 2.16 & 1.21 & 0.12 & 148 & 0.68 \\
\hline Triunfar.Piel & 2.09 & 1.08 & -0.16 & 143 & 0.70 \\
\hline Apodos & 1.74 & 1.44 & 0.98 & 160 & 0.62 \\
\hline Indito & 1.15 & 2.20 & 3.89 & 215 & 0.46 \\
\hline Gracioso.Apodos & 0.94 & 2.58 & 5.61 & 252 & 0.40 \\
\hline Morena.Violenta & 0.79 & 2.71 & 6.84 & 254 & 0.39 \\
\hline Insultar.Piel & 0.60 & 3.28 & 10.25 & 311 & 0.32 \\
\hline Inteligentes & 0.48 & 3.86 & 15.51 & 345 & 0.29 \\
\hline$\overline{\mathrm{XX}}=1.48 \quad$ Ss=0.66 LS=2.14 & LI $=.82$ \\
\hline
\end{tabular}

Tabla 3 Análisis univariable de racismo

Fuente: elaboración propia
Con respecto a sesgo, los valores se inclinan al positivo, lo que indica que tienen tendencia a la parte baja de la escala. La representación icónica de las variables indica que son leptocúrticas $(K>3)$, con tendencia de colas menos anchas en la representación de la normal, sin embargo, del análisis se pueden observar 4 datos con tendencia platicúrtica $(\mathrm{K}<3)$, los cuales son: es normal referirse como morenito a una persona de piel morena, es normal referirse a una persona de piel morena como prieto, es más fácil triunfar en la vida si tu color de piel es claro y es gracioso poner apodos a las personas por su color de piel. De acuerdo al puntaje $Z(>1.96)$ se lee que la tendencia es a no extrapolar, ya que tienen alta variabilidad $(K<1.96)$ en sus respuestas.

\section{Análisis Univariable de Estereotipos de Nivel económico}

Con base en el análisis de media de medias se observa que los sujetos indican que mayormente los estereotipos de clase consistentes en que las personas son pobres porque son flojas y las personas en situación de pobreza son más violentas que las personas ricas. De forma regular la gente en situación de pobreza viola más las leyes que las personas de clase alta.

En menor proporción, mencionan que está bien que a la gente pobre no la dejen entrar a ciertos lugares donde consumen la clase alta, los indígenas son pobres porque no les gusta trabajar y siento temor cuando una persona pobre está cerca de mí.

\begin{tabular}{|l|l|l|l|l|l|}
\hline \multicolumn{1}{|c}{$\mathbf{n = 2 4 4}$} & $\overline{\mathbf{X}}$ & \multicolumn{1}{c}{ Sk } & \multicolumn{1}{c|}{ K } & \multicolumn{1}{c|}{$\mathbf{C V}$} \\
\hline Pobre.Flojas & 2.25 & 1.06 & 0.21 & 126 & 0.79 \\
\hline Pobreza.Viol & 2.04 & 1.08 & -0.04 & 138 & 0.73 \\
\hline Pobre.Ley & 1.17 & 1.98 & 3.42 & 190 & 0.53 \\
\hline Pobres.Lug & 0.89 & 2.65 & 6.58 & 239 & 0.42 \\
\hline Indígenas.Pob & 0.83 & 2.60 & 6.53 & 237 & 0.42 \\
\hline Temor.Pobre & 0.69 & 3.03 & 9.38 & 262 & 0.38 \\
\hline$\overline{X X}=1.31 \quad$ Ss $=0.43$ LS $=1.74$ LI $=0.88$ \\
\hline
\end{tabular}

Tabla 4 Análisis univariable de estereotipo de nivel económico

Fuente: elaboración propia

Con respecto a sesgo, los valores se inclinan al positivo, lo que indica que tienen tendencia a la parte baja de la escala. 
La representación icónica de las variables indica que son leptocúrticas $(K>3)$, con tendencia de colas menos anchas en la representación de la normal, sin embargo, del análisis se pueden observar 2 datos con tendencia platicúrtica $(\mathrm{K}<3)$, los cuales son: las personas son pobres porque son flojas y las personas en situación de pobreza son más violentas que las personas ricas. De acuerdo al puntaje $Z(Z>1.96)$ se lee que la tendencia es a no extrapolar, ya que tienen alta variabilidad $(\mathrm{K}<1.96)$ en sus respuestas.

\section{Análisis Univariable de Machismo}

Con base en el análisis de media de medias se observa que los sujetos indican que mayormente están de acuerdo con los siguientes enunciados que construyen el machismo como: una función natural del hombre es proteger a las mujeres, los piropos de hombres a mujeres son agresiones y que, los hombres deben ser los proveedores económicos del hogar. Además, concuerdan con que los hombres controlan menos sus impulsos sexuales, las mujeres que trabajan con muchos hombres siempre son acosadas, se ven mal las mujeres que toman alcohol, las mujeres que tienen múltiples parejas sexuales son fáciles y que las mujeres provocan que los hombres les falten al respeto con su forma de comportarse.

De forma regular concuerdan que los hombres controlan menos sus emociones que las mujeres, las mujeres provocan que los hombres les falten al respeto con su forma de vestir, un hombre que es muy sensible es considerado afeminado y se ven mal las mujeres que se juntan con muchos hombres.

También de forma regular concuerdan con que, las adolescentes embarazadas son un mal ejemplo para las demás, se ven mal los hombres que se juntan con muchas mujeres, los profesores que se involucran sexualmente con alumnas es porque ellas los provocan y que todos los hombres son violentos. Con respecto a sesgo, los valores se inclinan al positivo, lo que indica que tienen tendencia a la parte baja de la escala. La representación icónica de las variables indica que son platicúrticas $(K<3)$, con tendencia de colas más anchas en la representación de la normal, sin embargo, del análisis se pueden observar 12 datos con tendencia leptocúrtica $(\mathrm{K}>3)$.
De acuerdo al puntaje $Z(Z>1.96)$ se lee que la tendencia es a no extrapolar, ya que tienen alta variabilidad $(K<1.96)$ en sus respuestas.

\begin{tabular}{|l|r|r|r|r|r|}
\hline \multicolumn{1}{|c}{$\mathbf{n = 2 4 4}$} & \multicolumn{1}{c}{ Sk } & \multicolumn{1}{c|}{ CV } \\
\hline H.Prot.Muj & 5.18 & -0.14 & -1.40 & 73 & 1.37 \\
\hline Piropos & 4.26 & 0.25 & -1.12 & 82 & 1.22 \\
\hline H.Provee & 3.80 & 0.29 & -1.23 & 92 & 1.08 \\
\hline H.Impu sex. & 3.44 & 0.42 & -1.17 & 99 & 1.01 \\
\hline M.Acosa & 3.06 & 0.49 & -0.87 & 97 & 1.03 \\
\hline M.Alcohol & 3.05 & 0.64 & -0.87 & 110 & 0.91 \\
\hline M.Fáciles & 2.88 & 0.83 & -0.63 & 119 & 0.84 \\
\hline M.Fal res.Co. & 2.75 & 0.74 & -0.77 & 117 & 0.85 \\
\hline H.Emociones & 2.45 & 0.88 & -0.35 & 122 & 0.82 \\
\hline M.Fal res.Ves & 1.91 & 1.23 & 0.16 & 152 & 0.66 \\
\hline Homb Afem & 1.82 & 1.39 & 0.83 & 153 & 0.65 \\
\hline M.ju Muc hom & 1.78 & 1.19 & 0.27 & 147 & 0.68 \\
\hline Adol.Emba & 1.66 & 1.62 & 1.72 & 165 & 0.61 \\
\hline H.Jun.MucMuj. & 1.63 & 1.53 & 1.23 & 167 & 0.60 \\
\hline Prof.sexu & 1.32 & 1.96 & 3.30 & 184 & 0.54 \\
\hline TodH.Viol & 1.30 & 1.62 & 1.49 & 179 & 0.56 \\
\hline Ado. Promi & 1.20 & 2.14 & 3.75 & 205 & 0.49 \\
\hline Papá permi & 1.14 & 1.92 & 2.76 & 201 & 0.50 \\
\hline Cuid hijos.M & 1.05 & 1.71 & 1.46 & 198 & 0.51 \\
\hline Hombre.llora & 0.90 & 2.47 & 5.33 & 239 & 0.42 \\
\hline M.Men fies & 0.89 & 2.23 & 4.20 & 225 & 0.44 \\
\hline Pegarían & 0.88 & 2.36 & 5.28 & 221 & 0.45 \\
\hline Ego.M.Div & 0.84 & 2.35 & 4.89 & 227 & 0.44 \\
\hline Muj de ver & 0.80 & 2.82 & 7.35 & 267 & 0.37 \\
\hline Esp.Hom & 0.79 & 2.65 & 6.84 & 247 & 0.40 \\
\hline Aut. de hom & 0.77 & 2.45 & 5.55 & 238 & 0.42 \\
\hline M.Bon.Acos & 0.74 & 2.82 & 7.42 & 262 & 0.38 \\
\hline M.Emb.dEsc. & 0.66 & 2.69 & 6.72 & 252 & 0.40 \\
\hline Esposa.Some & 0.64 & 3.29 & 10.80 & 290 & 0.35 \\
\hline XX 1.84 Ss= 0.62 & LS $=2.46$ & LI= 1.22 & \\
\hline
\end{tabular}

Tabla 5 Análisis univariable de machismo Fuente: elaboración propia

\section{Análisis Univariable de Violencia en el entorno escolar}

Con base en el análisis de media de medias se observa que los sujetos indican que mayormente son tipos de violencia que detectan en el entorno escolar la derivada de la preferencia sexual, homofobia, machismo y condición económica.

De forma regular la discriminación por color de piel y en menor proporción el sexismo, por embarazo y por orientación vocacional. 


\begin{tabular}{|c|c|c|c|c|c|}
\hline$n=244$ & $\bar{X}$ & Sk & $\mathbf{K}$ & CV & $\mathbf{Z}$ \\
\hline Pref sex & 4.46 & 0.01 & -1.24 & 76 & 1.32 \\
\hline Homofobia & 4.31 & 0.10 & -1.26 & 80 & 1.24 \\
\hline Machismo & 4.25 & 0.09 & -1.29 & 82 & 1.22 \\
\hline Codic econ & 4.15 & 0.09 & -1.16 & 78 & 1.28 \\
\hline Color piel & 3.67 & 0.29 & -1.12 & 88 & 1.13 \\
\hline Sexismo & 3.39 & 0.38 & -0.99 & 91 & 1.09 \\
\hline Embarazo & 3.33 & 0.45 & -1.01 & 95 & 1.05 \\
\hline Orien voc. & 2.61 & 0.73 & -0.59 & 113 & 0.89 \\
\hline
\end{tabular}

Tabla 6 Análisis univariable de violencia en el entorno escolar

Fuente: elaboración propia

Con respecto a sesgo, los valores se inclinan al positivo, lo que indica que tienen tendencia a la parte baja de la escala. La representación icónica de las variables indica que son platicúrtica $(\mathrm{K}<3)$, con tendencia de colas más anchas en la representación de la normal. De acuerdo al puntaje Z (>1.96) se lee que la tendencia es a no extrapolar, ya que tienen alta variabilidad $(\mathrm{K}<1.96)$ en sus respuestas.

\section{Conclusiones}

Con base en los resultados presentados para dilucidar el estado actual de la violencia simbólica medida a través de la orientación vocacional o currículum, estereotipos de sexualidad, racismo, nivel económico, machismo y la violencia per se en el entorno escolar se llega a las siguientes conclusiones.

Se afirma que con respecto a la orientación vocacional o currículum, el personal docente tiene percepciones estereotípicas respecto al rol social que deben desempeñar hombres y mujeres y su relación con la orientación vocacional, donde vinculan la profesión con el género o por preferencia sexual, construyendo ante eso una descalificación sexual a quienes eligen profesiones no tradicionales y etiquetando de forma violenta a quien transgrede dichos roles históricos, reforzando de esta manera la diferencia entre las oportunidades a que tendrá acceso el estudiantado con relación al campo público y privado de dichos individuos. En cuanto a los estereotipos de sexualidad se afirma que existe un rompimiento a la visión tradicional de la sexualidad al existir una apertura hacia la práctica de la sexualidad fuera de lo tradicionalmente aceptado, es decir, dentro del matrimonio, sin embargo sigue siendo más aceptado en el caso de los hombres.
Además, el profesorado muestra una invisibilización sobre los derechos sexuales de las personas adolescentes, al asumir que el sexo es para adultos, cuando la práctica de la sexualidad generación tras generación se ha normalizado entre grupos cada vez más jóvenes, por lo que, este rechazo invisibiliza la práctica sexual del estudiantado y la necesidad de orientación adecuada.

De racismo como parte de la violencia simbólica se concluye que el personal docente considera como normal el uso de peyorativos en el lenguaje, que se encuentran relacionados con el color de piel de las personas, en este caso, de las personas de tez morena, lo que constituye una forma de discriminación sutil, al diferenciar a los sujetos por sus características físicas.

También se puede inferir que, ligan el éxito de una persona con su color de piel y no por su inteligencia, habilidad o trayectoria, por lo que se percibe el factor clasista-racista dentro del pensamiento del profesorado que normalizan desde su construcción y que reproducen dentro de las estructuras sociales en las que participan.

Se concluye del estereotipo de nivel económico que la forma en que percibe el personal docente la pobreza, recae en elementos que promueven la construcción de estereotipos que repercuten en el estudiantado, ya que el personal docente al no tener control de quiénes son o serán sus estudiantes pueden tener en este sentido, colectivos que se encuentren en situación de pobreza, lo cual conlleva a que esta representación simbólica del profesorado hacia la misma, promueve la desigualdad de oportunidades por el clasismo a que son sujetos dichos colectivos y las formas de discriminación que esto trae consigo. 
Uno de los tópicos más relevantes en la violencia simbólica dentro de esta investigación; es lo que refiere al machismo, del cual se afirma que el profesorado mantiene las construcciones culturales machistas que promueven formas distintas de violencia hacia las mujeres, tales como las derivadas de caracterizar desde su concepción al varón como un ser humano con un rol estereotípico que lo visualiza como un ser proveedor, protector e incapaz de contener sus emociones sexuales, lo cual justifica las agresiones -ya sean las denominadas simbólicas, que escalan conforme al tiempo en psicológicas y físicas- en la sociedad, que se puede observar cuando normalizan el acoso y la violencia como una situación provocada por la víctima, en este caso, las mujeres y no por el victimario, en decir, los hombres; e inclusive concuerdan con los estereotipos de la libertad sexual como algo ligado a la calidad moral del ser humano, al elegir el enunciado con el concepto "fácil" para referirse a una mujer. Es importante prestar atención al enunciado sobre los profesores que se involucran sexualmente con estudiantes, puesto que este enunciado conlleva tres relaciones de poder simbólico machista, uno derivado de la relación vertical entre profesores y las estudiantes, otro derivado de que la construcción del enunciado que delimita a profesores varones con estudiantes mujeres y el último derivado de la relación entre un adulto con una estudiante de bachillerato, cuyas edades oscilan entre los 15-18 años. Aunque no en mayor escala, pero se sigue percibiendo que las emociones y la elección de grupos sociales es parte de lo que da poder a un sujeto, en este caso, se infiere que el profesorado tiene estereotipos respecto a las emociones y los grupos con los que un varón debe socializar.

Al ser el machismo una construcción donde el poder deriva de las pautas marcadas de lo que los hombres esperan de las mujeres para ser dignas de valor, el juntarse con mujeres significaría una transgresión que no debe ser promovida por lo que debe evitarse a través de diferentes mecanismos como lo es determinar el que "se verán mal" aquellos varones que decidan juntarse con mujeres (cabe señalar que viceversa, es decir, mujeres que se juntan con muchos hombres opera de la misma manera, ya que la construcción del machismo cultural no se concibe como una característica permitida sino como transgresora), lo mismo pasa con las emociones, las cuales han estado ligadas a la construcción cultural de característica femenina.
Finalmente, de la violencia per se se indica que las formas de discriminación que el personal docente observa en el entorno escolar incluye las derivadas de preferencias sexuales distintas, machismo, condición económica y color de piel, todas derivadas de grupos vulnerables que tradicionalmente carecen del mismo valor que aquellos que poseen el poder simbólico en la construcción social, como lo es el ser un hombre blanco, heterosexual y rico, lo cual constituye una intersección difícil de conseguir para todos los grupos en edad escolar bachiller de los distintos centros educativos, es decir, el profesorado percibe que la construcción del estereotipo mencionado es lo que provoca que aquellos que posean alguna característica valorada en la sociedad tradicional pueda agredir, violentar o discriminar a los que no la poseen, como el blanco frente al moreno, el rico frente al pobre, el hombre frente a la mujer y el heterosexual frente al homosexual.

\section{Referencias}

Aguilar Montes de Oca, Y. P., Valdez Medina, J., González Escobar, S., \& Gonzalez, N.-F. (2013). Los roles de género de los hombres y las mujeres en el México contemporáneo. Enseñanza e investigación en psicología VOL. $18,207-224$.

Abagnano, N. (1993). Diccionario de filosofía . México: Fondo de Cultura Económica.

Alcántara , A., \& Navarrete Cazal, Z. (2014). Inclusión, equidad y cohesión social en las políticas de educación superior en México. Revista mexicana de investigación educativa, 213-239.

Barreto, M. (2017). Violencia de género y denuncia pública. Revista mexicana de sociología, 261286.

Bourdieu, P. (2000). La dominación masculina. Barcelona: Anagrama.

Bourdieu, P., \& Passeron, J.-C. (2014). La reproducción. Elementos para una teoría del sistema de enseñanza (Tercera edición ed.). México: Fontamara.

Buquet, A., Araceli, M., \& Moreno, H. (2018). Imaginario occidental y expulsión de las mujeres de la. Revista de la Educación Superior 47, 83108. 
Comisión Interamericana de Derechos Humanos. (2013). Informe preliminar sobre el tema "Orientación sexual, identidad de género y expresión de género". Río de Janeiro.

Comisión Nacional de los Derechos Humanos. (02 de 11 de 2017). Obtenido de http://www.cndh.org.mx/Que_son_Derechos_H umanos

Comisión Nacional de los Derechos Humanos. (s.f.). Cartilla de Derechos Sexuales de Adolescentes y Jóvenes. Obtenido de 2016: http://www.cndh.org.mx/sites/all/doc/Programa s/VIH/Divulgacion/cartillas/CartillaDerechos-

Sexuales-Adolescentes-Jovenes.pdf

Congreso de la Unión. (1 de 05 de 2007). Ley General de Acceso de las Mujeres a una Vida Libre de Violencia. Obtenido de http://www.diputados.gob.mx/LeyesBiblio/pdf/ LGAMVLV_220617.pdf Congreso de la Unión, Cámara de Diputados. (2014). Ley General de los Derechos de Niñas, Niños y Adolescentes. México.

Consejo Nacional Para Prevenir la Discriminación. (2007). La Diversidad Sexual y Los Retos de la Igualdad y la Inclusión. Obtenido de http://www.conapred.org.mx/documentos_cedo c/E0005(1).pdf Consejo Nacional para Prevenir la Discriminación. (2016). Glosario de la diversidad sexual, de género y características sexuales. Ciudad de México. Obtenido de http://www.conapred.org.mx/documentos_cedo c/Glosario_TDSyG_WEB.pdf

Consejo Nacional Para Prevenir La Discriminación. (02 de 11 de 2017). Obtenido de http://www.conapred.org.mx/index.php?conteni $\mathrm{do}=$ pagina\&id=84\&id_opcion=142\&op=142

Constitución Política de los Estados Unidos Mexicanos. (s.f.). Camara de Diputados. Recuperado el 20 de 12 de 2016, de http://www.diputados.gob.mx/LeyesBiblio/pdf/ 1_150816.pdf

Convención sobre la eliminación de todas las formas de discriminación contra la mujer, CEDAW. (2011). MUJERES, ONU. Obtenido de

http://www2.unwomen.org//media/field\%20offi ce\%20mexico/documentos/publicaciones/2011/ convenci\%C3\%B3n\%20 pdf.pdf?la=es
De Beauvoir, S. (1949). El Segundo Sexo. Buenos Aires: Sudamericana.

DeChile. (2017). Diccionario Etimológico español en línea. Obtenido de http://etimologias.dechile.net/?percepcio.n

Declaración Universal de Derechos Humanos. (1948). Naciones Unidas. Obtenido de http://www.un.org/es/universal-declarationhuman-rights/

Espinoza, N. Á. (2016). La moral, los roles, los estereotipos femeninos y la. humanidades, 1-32.

Galarza Fernández, E., Cobo Bedía, M., \& Esquembre Cer, M. (2016). Medios y violencia simbólica. Revista Latina de Comunicación Social, 818-832.

Grollmus, N. S. (2012). La construcción social de la masculinidad: poder,hegemonía y violencia. Psicología, Conocimiento y Sociedad 2 (2), 27-65.

Gutiérrez, E. J. (2015). Códigos de masculinidad hegemónica en educación. Revista Iberoamericana de Educación, vol. 68 , 79-98.

Segato, R. L. (2003). Las estructuras elementales de la violencia: contrato y status en la etiología de la violencia. Obtenido de http://www.escuelamagistratura.gov.ar/images/ uploads/estructura_vg-rita_segato.pdf

Suprema Corte de Justicia de la Nación. (2014). Protocolo de actuación para quienes imparten justicia en casos que involucren la orientación sexual o la identidad de género. México.

UNICEF. (2006). Convención Sobre los Derechos Del Niño. Madrid.

Ursini, S. y. (2017). Equidad, género y matemáticas en la escuela mexicana. Revista Colombiana de Educación, N. ${ }^{\circ}$ 73, 213-234.

Yogyakarta, P. d. (12 de junio de 2018). Obtenido de www.yogyakartaprinciples.org/ 